\title{
Automatische Festpastenzufuhr steigert Produktivität
}

\author{
Ein neues Auftragsverfahren für feste Polierpasten kombiniert die Vorteile von Emulsionen und \\ Festpasten und sorgt für eine wirtschaftlichere Produktion. Eine Pilotanlage wurde beim Armaturen- \\ und Brausenhersteller Hansgrohe erfolgreich in Betrieb genommen.
}

Die Hansgrohe Group setzte beim automatisierten Polieren ihrer Messingarmaturen bisher ausschließlich auf Polieremulsion, doch die Produktivitätsreserven waren erschöpft. Festpasten garantieren im Vergleich zu Emulsionen eine höhere Oberflächenqualität, sind länger lagerfähig und Bearbeitungs- sowie Reinigungszeiten verkürzen sich erheblich. Allerdings war ein wirtschaftlicher Einsatz im automatisierten Prozess bislang nicht möglich. Mit dem Ziel Taktzeiten zu reduzieren, manuellen Aufwand zu senken und die Brillanz der Teile weiter zu erhöhen, stellt Hansgrohe eine Polieranlage auf das MeltVerfahren (Menzerna Liquefaction Technology) um. Diese Technologie ist ein von Menzerna in Zusammenarbeit mit SM Klebetechnik entwickeltes Festpasten-Auftragsverfahren für automatische Polieranlagen. Durch das Verfahren können Polieranlagen über lange Zeit automatisch mit Festpaste versorgt werden.

\section{Pastenmenge und Zuführintervall exakt einstellbar}

Die Anlage wurde in Kooperation mit SHL und SM Klebetechnik in eine bestehende Polieranlage von Hansgrohe integriert. Über ein 200-Liter-Fass werden zwei Polierstationen versorgt, die im Drei-Schichtbetrieb laufen. Eine erwärmte Druckplatte schmilzt die oberste Schicht der Paste im Fass auf. Die zähflüssige Paste wird von einer Pumpe über ein beheiztes Schlauchsystem zu einem Dosierkopf geleitet und dort auf den Polierring aufgetragen.

Der Dosierkopf ersetzt die Sprühpistole beziehungsweise das Festpastenzuführgerät und fährt im vorgegebenen Intervall an das Polierwerkzeug. Eine Verdrängerpumpe drückt die Polierpaste durch eine Schlitzdüse am Dosierkopf auf den Polierring. Zuführintervall, Pastenmenge und weitere Parameter lassen sich an der Steuerungseinheit exakt einstellen. Somit kön- nen erhebliche Produktivitäts- und Qualitätssteigerungen in automatisierten Polierverfahren erzielt werden. „Schon nach einer ersten groben Optimierung haben wir mit Melt eine Taktzeitreduktion im zweistelligen Prozentbereich erreicht", berichtet Jürgen Nähr, Technologe in der Poliererei bei der Hansgrohe Group im Werk Schiltach West.

Menzerna wird in Zusammenarbeit mit Hansgrohe weitere Optimierungen vornehmen, um das Verfahren noch wirtschaftlicher zu machen. Ein zusätzlicher Vorteil der Technologie: Der Verschmutzungsgrad in der Polierzelle ist deutlich geringer, was den Reinigungsaufwand erheblich reduziert. //

\section{Kontakt}

Menzerna Polishing Compounds

GmbH \& Co. KG, Ötigheim

Sven Bürkle, Leitung Marketing

Tel. 07222915770

sven.buerkle@menzerna.com

www.menzerna.de

einem 200-Liter-Fass

oberflächlich angeschmolzen

und über beheizte Leitungen

zu einem Dosierkopf am

Polierring gepumpt.

An einem Polierrad werden die Armaturen poliert.

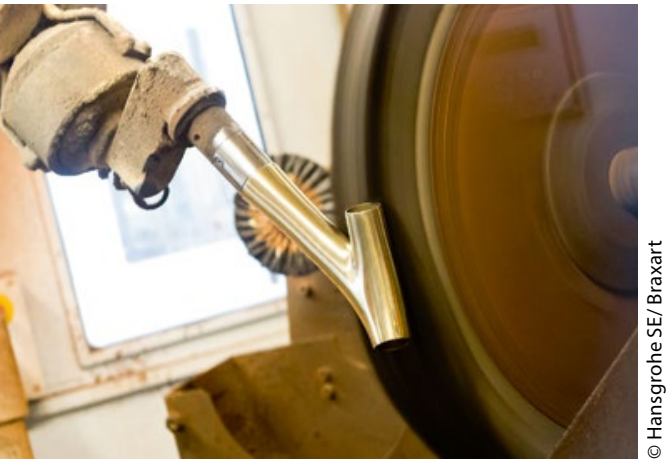

